\title{
Heilkräfte aus der Maschine - Elektrische und magnetische Kuren im 18. Jahrhundert*
}

Von Heinz Schott

\section{Zur Vorgeschichte}

Die Begriffe der Elektrizität und des Magnetismus verweisen etymologisch auf die Antike. Die anziehende Kraft des «Elektron», des geriebenen Bernsteins, und die des «magnetis lithos», des Magneteisensteins aus der Umgebung der kleinasiatischen Stadt Magnesia, wurden als analoge Erscheinungen betrachtet ${ }^{1}$. In der medizinischen Geschichtsschreibung wird üblicherweise auf die Verwendung tierischer Elektrizität in der Antike verwiesen: auf die schockartigen Kuren mit dem Zitterrochen (Torpedo ocellata und marmorata) und dem Zitteraal (gymnotus electricus) ${ }^{2}$. Ebenso bekannt ist die therapeutische Verwendung des Magneteisensteins in der Medizingeschichte, insbesondere bei Paracelsus.

Doch erst ab 1600 können wir in der Wissenschaftsgeschichte von Elektrizität und Magnetismus als voneinander abgrenzbaren Gegenstandsbereichen sprechen. Wegweisend war hier William Gilberts (1544-1603) Buch «De magnete magneticisque corporibus et de magno magnete Tellure physiologia nova» (1600), worin erstmals der Magnetismus wissenschaftlich dargestellt und eine pragmatische Methode zur Herstellung von Dauermagneten aus Stahl gegeben wurde ${ }^{3}$. 60 Jahre später konnte Otto von Guericke (1602-1686), der Bürgermeister von Magdeburg, mit einer Schwefelkugel einen elektrostatischen Generator konstruieren, der durch Reibung mit der Hand aufgeladen wurde ${ }^{4}$. Diese beiden Erfindungen von Gilbert und Guericke stehen am Anfang einer neuen Ära. In der Medizin jedoch spielen Magnetismus und Elektrizität im 17. Jahrhundert keine Rolle. Erst mit dem technischen Fortschritt, d.h. mit der Entwicklung von Elektrisiermaschinen und der Herstellung von starken Dauermagneten in der Mitte des 18. Jahrhunderts - im Zusammenhang mit dem Aufblühen der neurophysiologischen Forschung - interessierte sich die Medizin für ihre therapeutische

\footnotetext{
* Vortrag, gehalten auf der 69. Jahrestagung der Deutschen Gesellschaft für Geschichte der Medizin, Naturwissenschaft und Technik am 21.9.1986 in Hannover.
} 
Nutzung. Elektrische und magnetische Kuren kamen im Sinne einer physikalisch begründeten Medizin, der «Iatrophysik», in Mode. Elektrische und magenetische Kräfte (als «Imponderabilien») ${ }^{5}$ sollten nun mit Hilfe bestimmter Apparaturen auf den kranken Organismus übertragen werden.

\section{Zur elektrischen Kur}

Zwei neue Apparate prägten das Bild der elektrischen Kur: (a) die Elektrisiermaschine, die aus einem Glaszylinder bestand und mit einem Schwungrad angetrieben wurde, wodurch Reibungselektrizität erzeugt werden konnte; und (b) die «Leidener Flasche», die im Verbund mit dieser Elektrisiermaschine als Kondensator und Verstärker diente ${ }^{6}$. Die Möglichkeit einer Elektrotherapie wurde erstmals von dem Naturforscher Johann Gottlob Krüger (1715-1759) aus Halle anvisiert, in der Zeit nach Georg Ernst Stahls (1659-1734) und Friedrich Hoffmanns (1660-1742) Wirken in Halle ${ }^{7}$. In seiner 1745 verfaßten «Zuschrifft/An seine Zuhörer/Worinnen er Ihnen seine Gedanken von der Electricität Mittheilet» sagt er, man müsse «die Electricität unter die medicinische Hülffs-Mittel zehlen» ${ }^{8}$. Er formuliert dabei den für die elektrische Kur des 18.Jahrhunderts maßgeblichen Grundsatz, «daß durch die Electrification eines Menschen die Säfte flüßig gemacht, und die festen Theile in den Stand gesetzt würden, sich mit grösserer Lebhaftigkeit zusammen zuziehen». ${ }^{9}$

Als Begründer der Elektrotherapie wird heute jedoch Krügers Schüler Christian Gottlieb Kratzenstein (1723-1795) angesehen, der 1744 in seinem kämpferischen «Schreiben von dem Nutzen der Electricität in der Arzneywissenschaft» die «Electrification» der Kranken explizit «vor eine Panacee» ausgibt ${ }^{10}$. Im Einklang mit Krüger sieht er ihre Heilwirkung darin begründet, daß sie die Stauungen der Körpersäfte, vor allem die des Blutes, auflöse, indem sie Schwefel und Salzteilchen austreibe. Somit sei die «Electrification» angezeigt bei Dickblütigkeit, Kongestionen aller Art (wie Kopfschmerz, Schnupfen, Brustbeschwerden), bei Fiebern und sogar der Pest. Kratzenstein empfiehlt auch die «Cur der dicken Bäuche»: «Denn weil das Fett meistens aus schweflichten Theilen besteht, so wird der dicke Bauch bald schmeltzen müssen, wenn man dieselben durch Elektrification herausjagt.» Noch besser sei es freilich, so fügt er mit gewisser Ironie hinzu, «wenn man einen solchen dicken Bauch alle Tage ein paar Stunden [am Rad der Elektrisiermaschine] drehen läßt». ${ }^{11}$ 
In den nachfolgenden Jahren befaßten sich zahlreiche Naturforscher und Ärzte mit der Elektrotherapie, wie z.B. Jean Antoine Nollet (1700-1770), Jean Jallabert (1712-1768), Karl von Linné (1707-1778), Albrecht von Haller (1708-1777), Anton de Haen (1704-1776), Jean Paul Marat (1749-1809) ${ }^{12}$ und - last but not least - Benjamin Franklin (1706-1790), der Heilversuche anstellte ${ }^{13}$. Übrigens erscheint die von ihm entwickelte Glasharmonika, die dann Mesmer bei seinen magnetischen Kuren zur Verstärkung des «Fluidums» einsetzte, als ein musikalisches Analogon oder Symbol der Elektrisiermaschine.

Weniger bekannt wurde der Regensburger Arzt Johann Gottlieb Schäffer (1720-1795), der in seinem Lehrbuch «Die Electrische Medicin» von 1752 (2.Aufl. 1766) den theoretischen und praktischen Stand der zeitgenössischen Elektrotherapie differenziert dargestellt hat ${ }^{14}$. Das «electrische Seculum» schien Schäffer wie den meisten seiner Zeitgenossen angebrochen. Im Vorwort zur ersten Auflage bemerkt er: «es ist in der That wahr, die Luft, die Menschen, und fast alle Weltkörper, sind zu mancher Zeit electrisch. Ist es mir also wohl zu verdenken, wenn auch ich in dieser electrischen Zeit, gegenwärtige Schrift von dieser Materie zu schreiben, mich unterfangen habe?»

Wie Kratzenstein hält Schäffer die Kongestionen des Blutes durch die elektrische Kur für heilbar. Hauptindikation sind für ihn jedoch die «gelähmten Glieder». Angriffspunkte des Elektrisierens sind Muskeln und Nerven, welche alle Körperbewegungen verursachen. Dabei verhalte sich, so Schäffer, der Muskel zum Nerven wie das Rad einer Maschine zur Antriebskraft, welche dem «Nervensaft» oder «Nervengeist» entspreche. Die Elektrotherapie wird somit neurophysiologisch begründet: «was der Nervensaft natürlicherweise durch seinen Einfluß in die Muskeln thut; das verrichtet die Electricität auf eine künstliche Art, und dieses alles um so mehr, weil die electrische Materie in vielen Stücken mit dem Nervensaft viele Ähnlichkeit und fast einerley Eigenschaft zu besitzen scheint.» ${ }^{15}$

Interessant ist Schäffers Vorschlag, die Elektrizität auch innerlich, den «innerlichen Theilen des menschlichen Körpers» in Form einer «electrischen Arzney» beizubringen. Wasser, Wein und Tee könnten leicht elektrisiert und dem Patienten dargereicht werden, wobei der elektrisierte Wein «einen viel stärkeren Geruch von sich giebt, auch eher berauschet, als ein unelektrisierter» ${ }^{16}$. Ähnliches finden wir dreißig Jahre später im Mesmerismus wieder, wo z. B. «magnetisches» Wasser als Lebenselixier getrunken wird. 


\section{Zur magnetischen Kur}

Die magnetische Kur, d.h. die Kur mit Eisenmagneten - nicht die des «tierischen Magnetismus», worauf wir noch zurückkommen werden entwickelte sich im 18. Jahrhundert gleichsam im Windschatten der elektrischen Kur. Die Anwendung neu entwickelter Dauermagnete ${ }^{17}$ war beileibe nicht so spektakulär wie das elektrische Szenarium mit Blitz, Funken und künstlichem Heiligenschein ${ }^{18}$, sowie den eindrücklichen Sensationen und sichtbaren Spuren der elektrischen Einwirkung auf der Haut ${ }^{19}$. Mitte des 18. Jahrhunderts sprach man zwar vom elektrischen, aber nicht vom magnetischen Zeitalter. Die Magnetwirkung schien sanfter, unmerklicher und weniger sensationell vonstatten zu gehen. Mit einer Phasenverschiebung von etwa 20 Jahren gegenüber der elektrischen Kur kam schließlich auch die magnetische um 1775 in Mode ${ }^{20}$.

Gegenüber der Elektrotherapie, die erst im 18. Jahrhundert entstand, stellt die Magnettherapie eine traditionelle Heilweise dar, die insbesondere der paracelsischen Tradition ihre Popularität in der Neuzeit verdankt. Bei Paracelsus mischen sich noch die verschiedenen Auffassungen: neben der rationalen Anwendung des Magneten beim Herausziehen von eisenhaltigen Fremdkörpern finden wir auch die spekulative Zentrierung deplazierter Körperorgane und -säfte sowie magische Formen von Krankheitszauber, wie etwa die «transplantatio morborum», die Krankheitsübertragung ${ }^{21}$. Diese wurde sogar in der Schule Charcots noch einmal aktuell: z. B. als Übertragung einer neurologischen Störung mit dem Hufeisenmagneten von einer Körperhälfte auf die andere ${ }^{22}$.

Während die Elektrizität offensichtlich aufrüttelnd und irritierend wirkte, schien der Magnetismus beruhigend, einschläfernd und vor allem schmerzstillend zu wirken ${ }^{23}$. So wurden in den 60er Jahren des 18. Jahrhunderts zunächst Zahnschmerzen ${ }^{24}$, bald aber auch Rheumatismus, Muskelverspannungen, Krämpfe, Angstzustände, Blindheit und Taubheit einer magnetischen Kur unterzogen. Ziehen, Wärme, Beruhigung, Schmerzstillung, Schweißabsonderung und Stuhlgang wurden als Heilwirkungen hervorgehoben ${ }^{25}$.

1774 schloß sich auch Franz Anton Mesmer (1734-1815) der in Mode gekommenen Magnetkur an und propagierte nach entsprechenden Erfolgen diese Heilmethode. Auf sein Anraten führte der Hamburger Arzt Johann Christoph Unzer (1747-1809) eine magnetische Kur durch, die ich als Fallbeispiel wiedergeben möchte. Wir stoßen hier auf dasselbe Arrange- 
ment, wie es Mesmer schon bei seiner Behandlung der «Jungfer Österlin» geschildert hat ${ }^{26}$. Es handelte sich um eine Frau von 26 Jahren, die seit ihrem 13. Lebensjahr ständig gekränkelt hatte. Nach ihrer vierten Niederkunft litt sie an Ohnmachten, Zittern, Krämpfen, Kopfschmerzen, rechtsseitiger Blindheit und Lähmung der Gliedmaßen, Konvulsionen und Schmerzen. «Es wurde ein Magnet an den Knöchel der rechten Hand und einer auf jede Wade gebunden; danach Ziehen vom Kopf nach der Schulter bis zu den Fingern, Gefühl von Wärme und Brennen. Nach 10 Stunden war der Kopf beweglicher und nach drei Tagen waren die meisten sichtbaren Zufälle der Krankheit gehoben! Vom 5. Tage ab Stuhlgang regelmäßig, ebenso Urinabsonderung, und reichlicher Schweiß. Am 12. Tage beginnende Appetenz nach Eintritt der Menses. Am 20. Tage verließ die Kranke erstmals das Bett.» ${ }^{27}$

Im Jahre 1779 legten Andry (1741-1829) und Thouret (1748-1810) im Auftrage der Société Royale de Médecine einen umfassenden Untersuchungsbericht über den Gebrauch des Magneten in der Heilkunde vor, der ausführlich über die magnetische Kur im 18. Jahrhundert informiert. Nach Darlegung einer reichhaltigen Kasuistik kommen die Autoren zum Schluß, daß der Magnetismus eine heilsame Wirkung auf den Körper ausübe, daß er dabei direkt auf die Nerven wirke und deshalb vor allem bei Nervenkrankheiten, wie etwa Krämpfen, Konvulsionen und Neuralgien, anzuwenden sei ${ }^{28}$. Magnetische Armreifen, Amulette, Gürtel und Platten, deren Konstruktion und Anwendung detailliert angegeben wurden, erfreuten sich allgemeiner Beliebtheit ${ }^{29}$. Sie erinnern an die Apparate der «Metallotherapie» im 19. Jahrhundert, worauf wir noch zurückkommen werden.

\section{Mesmerismus und Perkinismus als spektakuläre Abwandlungen}

Die elektrischen und magnetischen Kuren zwischen Barock und Aufklärung bildeten den Nährboden für spektakuläre Abwandlungen, wie wir sie zum einen beim «tierischen Magnetismus» oder Mesmerismus, zum anderen beim Perkinismus studieren können ${ }^{30}$. Franz Anton Mesmer (1734-1815) war durch seine Zugehörigkeit zur Wiener Fakultät bestens informiert über die zeitgenössische Elektrotherapie, die er vermutlich ab 1766 auch in seiner eigenen Praxis einsetzte. Ebenso war er - ab 1774 - durch seine Bekanntschaft mit dem Wiener Hofastronomen Pater Hell (1720-1792) auch über den neuesten Stand der Magnettherapie informiert, die er selbst mit Erfolg 
anwandte und nachdrücklich propagierte. Aber schon bald trug Mesmer seine These vom «tierischen Magnetismus» vor, mit der er der Schulmedizin eine neue Grundlage schaffen wollte: es gebe, so entwickelte er im einzelnen in seinen 27 Lehrsätzen von 1775, ein universelles Fluidum («Allflut»), eine viel feinere physikalische Substanz als diejenige, die von der Elektrizität und dem Magnetismus ausgehe ${ }^{31}$. Dieses heilsame Agens, das er «tierischen Magnetismus»(Lebensmagnetismus) nannte, könne über das Nervensystem auf den kranken Menschen übertragen werden, und zwar durch bestimmte Manipulationen wie Handauflegen, Striche oder durch den «baquet magnétique», den «Gesundheitszuber».

Dieser höchst spektakuläre Heilapparat kombinierte in seinem Aufbau sowohl elektrische als auch magnetische Elemente. Dies läßt sich am letzten erhaltenen Baquet aus der Mesmer-Zeit genauer studieren, der heute im Medizinhistorischen Museum der Universität Lyon zu besichtigen ist ${ }^{32}$. Dieser Baquet enthält Magnete, eine Leidener Flasche und ein elektrisches System und ist mit Metalleitern, Wollstricken und organischen (isolierenden) Substanzen ausgestattet. Der Clou von Mesmers Heilsystem war jedoch, daß die Heilkraft nicht identifiziert wurde mit Elektrizität und Magnetismus, wenngleich sie durch elektrische und magnetische Faktoren verstärkt werden konnte. Die wissenschaftlichen Untersuchungskommissionen in Paris schrieben die Wirkung der mesmeristischen Kur der «Imagination»zu, und die Gegner verurteilten Mesmer als Mystizisten und Scharlatan. Studiert man jedoch Mesmers eigene Schriften, so erweist sich dieser paradoxerweise als ein Kind der Aufklärung, als Anhänger einer physikalischen Medizin. Auf die spezifisch romantische Modifikation seines Systems werden wir noch einzugehen haben.

Im Jahre 1790, 15 Jahre nach Mesmers «Entdeckung», leitete Luigi Galvani (1737-1798) mit seinen experimentellen Untersuchungen zur «animalischen Elektrizität» eine neue Epoche in der Geschichte der Elektrotherapie ein, die als Galvanismus bezeichnet wird ${ }^{33}$. Wie Alessandro Volta (1745-1827) um 1800 herausstellte, kann die Verbindung zweier verschiedener Metalle galvanische Elektrizität erzeugen. Diese wurde nun in der Voltaschen Säule, einer Art Batterie aus Silber und Zink, gespeichert und konnte durch Elektroden auf kranke Organe abgeleitet werden.

Im Kontext des Galvanismus entfaltete sich eine weitere spektakuläre Heilmethode: der sogenannte Perkinismus ${ }^{34}$. Der amerikanische Arzt Elisha Perkins (1741-1799) entwickelte 1795 den «metallic tractor», eine zirkelförmige Gabel aus Messing und Eisen, die mit den Spitzen über die 
erkrankten Stellen gestrichen wurde ${ }^{35}$. Er ließ sich seine Erfindung patentieren und brachte sie dann serienmäßig in den Handel. Im Gegensatz zu Mesmer beriefen sich Elisha Perkins und dessen Sohn Benjamin (17741810), der seine Nachfolge antrat, auf die (galvanische) Elektrizität. Die «metallic tractors» sollten direkt die animalische Elektrizität, die sich in den kranken Körperteilen aufstaute, ableiten und entladen. Zur Hauptindikation des Perkinismus zählten dementsprechend Schmerz-, Entzündungs- und Fieberzustände. Die beiden Perkins ereilte dasselbe Schicksal wie den weit berühmteren Mesmer: die unbestreitbaren Heilerfolge wurden auf die Imagination zurückgeführt, womit ihre Heilmethode wissenschaftlich widerlegt schien.

\section{Zur Entwicklung im 19. Jahrhundert}

Alle Kuren, die wir vorgestellt haben, finden im 19. Jahrhundert ihre Fortsetzung.

(a) Die Begründung des Elektromagnetismus durch Michael Faraday (1791-1861) um 1830 löste den Galvanismus ab und initiierte die Elektrotherapie im modernen Sinne, insbesondere als eine neurologische und psychiatrische Behandlungsmethode, wie sie Duchenne de Boulogne (1806-1875) ab 1847 in Frankreich einführte ${ }^{36}$. Reibungselektrizität, Leidener Flasche und Galvanismus gehörten damit der Vergangenheit an.

(b) Demgegenüber setzte sich die Magnettherapie im 19. Jahrhundert ungebrochen fort. So empfahl René Théophile Hyacinthe Laennec (1781-1826) in seinem Hauptwerk «De l'auscultation médiate» die Anwendung des Magneten bei nervösen Brustbeschwerden, Asthma und Angina pectoris. Selbst beim Schluckauf helfe der Magnet: «Durch zwei magnetisirte Platten, von denen eine auf die Herzgrube und die andere gegenüber auf die Wirbelsäule gelegt wurde, habe ich Schlucksen, was bereits drei Jahre dauerte, in einem Augenblicke gehoben.» ${ }^{37}$

(c) Der «tierische Magnetismus» mit den verschiedensten Variationen des magnetischen Gesundheitszubers ${ }^{38}$ wandte sich unter dem Vorzeichen der Romantik dem Somnambulismus zu. Nicht mehr die magnetischen Manipulationen, sondern die wundersamen Eröffnungen und Offenbarungen der Somnambulen rückten nun in den Mittelpunkt des Interesses. Wir könnten hier in der Tat - 100 Jahre vor Viktor von Weizsäckers Überlegungen zur anthropologischen Medizin - von einer «Einführung des Subjekts» in die Medizin sprechen. 
(d) Der Perkinismus schließlich, die Anwendung von Metallstäben zu Heilzwecken, fand eine interessante Parallele in der Entfaltung der Akupunktur bzw. Elektropunktur («electro-puncture») im frühen 19. Jahrhundert ${ }^{39}$. Am auffälligsten jedoch knüpfte die «Metallotherapie» des französischen Arztes Victor Burq (1823-1884) um 1850 an die Tradition des 18. Jahrhunderts an, die alle bisher genannten Konzepte implizierte ${ }^{40}$. Burq glaubte, die individuell unterschiedliche physiologische Wirkung verschiedener Metalle entdeckt zu haben, wobei er vor allem dem Kupfer und seinen Legierungen (Messing und Bronze) besondere Kräfte zuschrieb. Er empfahl sie u. a. als «treffliche Schutzmittel» gegen die Cholera. Anne Harrington hat kürzlich darauf hingewiesen, daß Burq zu den Forschungen der CharcotSchule über Hysterie und Hypnose wohl den entscheidenden Anstoß gegeben hat, bei deren Experimenten Metalle und Magnete als Instrumente der Krankheitsübertragung dienten ${ }^{41}$.

Im 18. Jahrhundert wurden die Weichen für die therapeutische Nutzung der Elektrizität und des Magnetismus gestellt. Im 19. Jahrhundert kam es zu einer breiten Entfaltung der verschiedenen Ansätze. Wenngleich die meisten von ihnen von der Wissenschaft wieder verworfen wurden, leben sie seither zum Teil untergründig in der sogenannten Paramedizin weiter. Ich erinnere an Kupferarmbänder und Magnetmedaillons, die heute zum Kauf angeboten werden, und an Elektroakupunktur und Orgonakkumulatoren, die sogar von manchen Ärzten in Hinterzimmern betrieben werden. Der Internist Arthur Jores hat einmal darauf verwiesen, da $\beta$ auch die moderne Medizin nicht frei von Magie und Zauber sei. Mit seiner Bemerkung möchte ich schließen: «Eine bekannte Erscheinung ist der (gläubige> Patient vom Lande, der sich nach einer rein diagnostischen Untersuchung, z. B. der magisch so überaus wirksamen Röntgenuntersuchung, bei seinem Arzt dafür bedankt, wie gut ihm dies geholfen habe.» ${ }^{42}$

\section{Anmerkungen}

1 Vgl. Schmid, 1942, S. 83; bzw. Waldmann, 1878, S. 323.

2 Vgl. Schmid, 1942, S. 73 ff. Die elektrischen Organe des Zitterrochens wurden erst in der zweiten Hälfte des 18. Jahrhunderts anatomisch beschrieben und im Sinne der Elektrizitätslehre gedeutet; vgl. Anthony, 1977, S.635. Die «elektrischen Fische» wurden seinerzeit in Europa zur Schau gestellt und noch im 19. Jahrhundert in Kolonialländern therapeutisch eingesetzt; vgl. Kellaway, 1946, S. $133 \mathrm{ff}$. 
3 Vgl. Andrade, 1957, S.23f.

4 Vgl. Schmid, 1942, S. 84.

5 Vgl. Feldt, 1985.

6 Siehe Berninger, 1972. Dieses ursprüngliche Arrangement der elektrischen Kur wurde erst Ende des 18. Jahrhunderts vom Galvanismus und seiner Weiterentwicklung durch Volta geändert; vgl. Schmid, 1942, S. 106.

7 Vgl. Kaiser, 1977; Snorrason, 1974.

8 Siehe Krüger, 1745, S. 45.

9 Siehe Krüger, 1745, S. 47.

10 Siehe Kratzenstein, 1746.

11 Siehe Kratzenstein, 1746, S. 18.

12 Vgl. u. a. Schmid, 1942, S. 89 ff.; Mildner, 1967.

13 Vgl. Anthony, 1977, S. 636; Rogoff, 1969.

14 Siehe Schäffer, 1752.

15 Siehe Schäffer, 1752, S. 76.

16 Siehe Schäffer, 1752, S. 78.

17 Vgl. Andrade, 1957.

18 «Will man den Schein der Heiligen vorstellen, so darf man nur eine Haube, welche mit goldenen Tresen besetzt ist, aufsetzen, wenn man alsdann an einem Orte an der Haube Funcken herausfahren lässet, so werden rings um den gantzen Kopf herum, aus allen Öffnungen der Haube, Strahlen, herausfahren. Ich erinnere mich, daß schon 2 Frauenzimmer auf diese Art zu Heiligen gemacht worden» (siehe Schäffer, 1746, S.28).

19 Schäffer setzt die aufreizenden Mittel, wie sie traditionellerweise «wider die gelähmten Glieder» verwandt wurden, mit der Wirkung der Elektrizität gleich; vgl. Schäffer, 1752, S. $72 \mathrm{ff}$.

20 Vgl. hierzu vor allem die Kasuistik im zweiten Abschnitt von Andry und Thouret, 1785, S. 95-225; ebenso Heinsius, 1776.

21 Siehe Waldmann, 1878, S. $395 \mathrm{ff}$.

22 Vgl. Harrington, 1986.

23 Vgl. Waldmann, 1878, S. $430 \mathrm{f}$.

24. Vgl. z.B. Teske, 1765.

25 Vgl. Andry und Thouret, 1785.

26 Vgl. Mesmer, 1781, S. $13 \mathrm{ff}$.

27 Unzer (1775), zit. nach Waldmann, 1878, S.411.

28 Vgl. Andry und Thouret, 1785, S. $278 \mathrm{ff}$.

29 Vgl. Andry und Thouret, 1785, S.282-292.

30 Vgl. Carlson und Simpson, 1970; Quen, 1975.

31 Vgl. Mesmer, 1781.

32 Vgl. Enselme und Berger, 1966; Génard, 1982.

33 Vgl. Becquerel, 1857; Anthony, 1977.

34. Vgl. Herholdt und Rafn, 1798; Quen, 1975.

35 Vgl. Waldmann, 1878, S. 433.

36 Vgl. Ziemssen, 1857; Stainbrock, 1948, S. 159 ff.; Harms, 1954/55, S. 934; Schliephake, 1969, S.51. Es sei hier auf die umfassendste Bibliographie zur Geschichte der Elektrotherapie von Licht (1959) verwiesen sowie auf die sehr geraffte Übersicht von Turrell (1969). 
37 Zit. nach Waldmann, 1878, S.419.

38 Vgl. Kieser, 1818; Wolfart, 1818 und 1819; Kerner, 1829, S. 181-192.

39 Vgl. Stainbrock, 1948, S. 158; Quen, 1975.

40 Vgl. Burq, 1854.

41 Vgl. Harrington, 1986.

42 Siehe Jores, 1955, S.918.

\section{Literatur}

Andrade, E. N. da C.: Zur Frühgeschichte des Dauermagneten. In: Endeavour 16 (1957), S. $22-30$.

Andry, Charles Louis François, und Michel Augustin Thouret: Beobachtungen und Untersuchungen über den Gebrauch des Magnets in der Arzneykunst. Leipzig: Weidmann \& Reich, 1785.

Anthony, Nicholas: Electrical apparatus used in medicine before 1900. In: Proceedings of the Royal Society of Medicine 70 (1977), S.635-641.

Becker, Christian August: Der mineralische Magnetismus und seine Anwendung in der Heilkunst. Mühlhausen: Heinrichshofen, 1829.

Becquerel, A.: Traité des applications de l'électricité a la thérapeutique médicale et chirurgiale. Paris: Baillière, 1857.

Berninger, Ernst: Medicina electrica - Titelkupfer - (Rubrik: Unser Bild). In: Medizinhistorisches Journal 7 (1972), S.326-329.

Burq, V.: Metallotherapie. Behandlung der Nerven-Krankheiten, Paralysen, chronische Rheumatismen ... Leipzig: Schäfer, 1854.

Carlson, Eric T., und Meribeth M.Simpson: Perkinism vs. mesmerism. In: Journal of the history of the behavioural sciences 6 (1970), S. 16-24.

Enselme, J., und M.Berger: Le Baquet de Mesmer. In: Revue Lyonnaise de médecine 15 (1966), S.909-920.

Feldt, Heinrich: Vorstellungen von physikalischer und psychischer Energie zur Zeit Mesmers. In: Franz Anton Mesmer und die Geschichte des Mesmerismus. Beiträge zum Internationalen Wissenschaftlichen Symposion ... 1984 in Meersburg. Herausgegeben von Heinz Schott. Stuttgart: Steiner, 1985; S.31-43.

Génard, Philippe: D’un baquet magnétisme à l'histoire du Mesmerisme. Diss. Lyon, 1982.

Harms, Ernest: The origin and early history of electrotherapy and electroshock. In: The American journal of psychiatry 111 (1954/55), S.933-934.

Harrington, Anne: Metals and magnets in medicine. Hysteria, hypnosis, and scientific culture in late 19th-century France. Oxford, 1986 (unveröffentlichtes Typoskript, 22 S.).

Heinsius, Johannes Augustus: Beyträge zu den Versuchen mit künstlichen Magneten. Leipzig: Aue in Altona, 1776.

Herholdt, Johann Daniel, und Carl Gottlob Rafn: Von dem Perkinismus, oder den Metallnadeln des D. Perkins in Nordamerika ... Kopenhagen: Brunner, 1798.

Jores, Arthur: Magie und Zauber in der modernen Medizin. In: Deutsche Medizinische Wochenschrift 80 (1955), S.915-920. 
Kaiser, Wolfram: Johann Gottlob Krüger (1715-1759) und Christian Gottlieb Kratzenstein (1723-1795) als Begründer der modernen Elektrotherapie. In: Zahn-, Mund- und Kieferheilkunde 65 (1977), S. 539-554.

Kellaway, Peter: The part played by electrical fish in the early history of electricity and electrotherapy. In: Bulletin of the history of medicine 20 (1946), S. 112-137.

Kerner, Justinus: Die Seherin von Prevorst. Eröffnungen über das innere Leben des Menschen und über das Herinragen einer Geisterwelt in die unsere. 2 Theile. Stuttgart: Cotta, 1829.

Kieser, D.G.: Das Magnetische Behältniß (Baquet) und der durch dasselbe erzeugte Somnambulismus. Nach Theorie und Erfahrung. In: Archiv für den Thierischen Magnetismus 3 (1818).

Kratzenstein, Christian Gottlieb: Physikalische Briefe. 3. und 4. vermehrte Auflage. Halle: Hemmerde, 1746. (Darin: Schreiben von dem Nutzen der Electricität in der Arzneywissenschaft an D.G.F.F. 17.3 .1744 , S.1-20; Zweytes Schreiben von eben derselben Materie (7.10.1744〉, S.21-29.)

Krüger, Johann Gottlob: Zuschrifft / An seine Zuhörer / Worinnen er Ihnen seine Gedanken von der Electricität mittheilet und Ihnen zugleich seine künftige Lectionen bekannt macht. Neue und mit Anm. vermehrte Auflage. Halle: Hemmerde, 1745.

Licht, Sidney: History of Electrotherapy. In: Therapeutic Electricity and Ultraviolet Radiation. Ed. by Sidney Licht. New Haven: Elizabeth Licht, 1959 (Physical Medicine Library; 4); S. 1-69.

Mildner, Th.: Elektrotherapie vor 200 Jahren. In: Medizinische Klinik 62 (1967), S.352-355.

Mesmer, Franz Anton: Abhandlung über die Entdeckung des thierischen Magnetismus. Aus dem Französischen übersetzt. Carlsruhe: Macklot, 1781.

Quen, Jacques M.: Case studies in nineteenth century scientific rejection: Mesmerism, Perkinsism, and acupuncture. In: Journal of the history of the behavioral sciences 11 (1975), S. 149-156.

Rogoff, Joseph B.: B. Franklin on electrotherapy. In: The New England journal of medicine $280(1969)$, S. 673.

Schäffer, Johann Gottlieb: Die Kraft und Wirkung der Electricitet in dem menschlichen Körper und dessen Krankheiten besonders bey gelähmten Gliedern ... Regensburg: Bader, 1752 (2. Aufl. Regensburg 1966 unter dem Titel: Die Electrische Medicin oder die Kraft und Wirkung der Electricität ...).

Schliephake, E.: Zur Geschichte der Elektrotherapie. In: Archiv für physikalische Therapie 21 (1969), S.51-55.

Schmid, Alfred: Zur Geschichte der Elektrotherapie vom Altertum bis zum Beginn des 19. Jahrhunderts. In: Festschrift für Jacques Brodbeck-Sandreuter zu seinem 60. Geburtstag. Basel «1942〉.

Schneck, Jerome M.: The history of electrotherapy and its correlation with Mesmer's animal magnetism. In: The American journal of psychiatry 116 (1959/60), S.463-464.

Snorrason, E.: C.G. Kratzenstein ... and his experimental Studies on Electricity during the Eighteenth Century. Odense: University Press, 1974 (Acta historica scientiarum naturalium et medicinalium; 29).

Stainbrock, Edward: The use of electricity in psychiatric treatment during the nineteenth century. In: Bulletin of the history of medicine 22 (1948), S.156-177. 
Teske, Johann Gottfried: Versuch im Kuriren der Zahnschmerzen mittelst magnetischen Stahls. Königsberg: Hartung, 1765.

Turrell, W. J.: Landmarks of electrotherapy. In: Archives of physical medicine and rehabilitation 50 (1969), S.157-160.

Unzer, Johann Christoph: Beschreibung eines mit den künstlichen Magneten angestellten medicinischen Versuchs, Hamburg: Herold, 1775.

Waldmann, Wilhelm: Der Magnetismus in der Heilkunde. Eine Studie. In: Deutsches Archiv für Geschichte der Medicin und Medicinische Geographie 1 (1878), S. 320-355 u. S. 381-436.

Wolfart, Karl Christian: Einiges in Betreff des gemeinsamen Mesmerischen Leitungsbehältnisses. In: Jahrbücher für den Lebens-Magnetismus oder Neues Askläpieion 1 (1818) 1. Heft, S. 194-197; Fortsetzung in dieser Zeitschrift 1 (1819), 2. Heft, S.1-30.

Ziemssen, Hugo: Die Electricität in der Medicin. Studien. Berlin: Hirschwald, 1857.

\section{Summary}

\section{Healing powers by machines: electric and magnetic cures in the 18th century}

During the 18th century the scientific development of magnetism and electricity had a strong impact on medical theory and practice. Special equipments and "machines" were used to induce healing powers into the patient's nervous system. The transference of a supposed physical energy (electricity, magnetism, "animal magnetism" etc.) was managed by different therapeutic arrangements and techniques which were modified during the 19th century (e.g. galvano-magnetism, metallotherapy) and are still accepted in some aspects by adherents of "alternative medicine" nowadays.

Prof. Dr. Dr. Heinz Schott

Institut für Geschichte der Medizin der Universität Freiburg

Stefan-Meier-Straße 26

D-7800 Freiburg 